University of Nebraska - Lincoln

DigitalCommons@University of Nebraska - Lincoln

Peter Dowben Publications

Research Papers in Physics and Astronomy

January 2004

\title{
Are half-metallic ferromagnets half metals? (invited)
}

Peter A. Dowben

University of Nebraska-Lincoln, pdowben@unl.edu

Ralph Skomski

University of Nebraska-Lincoln, rskomski2@unl.edu

Follow this and additional works at: https://digitalcommons.unl.edu/physicsdowben

Part of the Physics Commons

Dowben, Peter A. and Skomski, Ralph, "Are half-metallic ferromagnets half metals? (invited)" (2004). Peter Dowben Publications. 7.

https://digitalcommons.unl.edu/physicsdowben/7

This Article is brought to you for free and open access by the Research Papers in Physics and Astronomy at DigitalCommons@University of Nebraska - Lincoln. It has been accepted for inclusion in Peter Dowben Publications by an authorized administrator of DigitalCommons@University of Nebraska - Lincoln. 


\title{
Are half-metallic ferromagnets half metals? (invited)
}

\author{
P. A. Dowben and R. Skomski \\ Department of Physics and Astronomy and the Center for Materials Research and Analysis (CMRA), \\ Behlen Laboratory of Physics, University of Nebraska, P.O. Box 880111, Lincoln, Nebraska 68588-0111
}

(Presented on 9 January 2004)

\begin{abstract}
Several classes of materials are currently under investigation as potential high-spin-polarization materials. Unfortunately, the proposed half-metallic materials, including the semi-Heusler alloys, the manganese perovskites, and the "simpler" oxides such as chromium dioxide and magnetite, suffer from fundamental limitations. First, the postulated half-metallic systems lose their full ( $T$ $=0)$ spin polarization at finite temperatures and, second, surfaces, interfaces, and structural inhomogenities destroy the complete spin polarization of half-metallic systems even at zero temperature. In a strict sense, half-metallic ferromagnetism is limited to zero temperature since magnon and phonon effects lead to reductions in polarization at finite temperatures. (C) 2004 American Institute of Physics. [DOI: 10.1063/1.1682911]
\end{abstract}

\section{INTRODUCTION}

By definition, the half-metallic ferromagnets have only one spin channel for conduction at the Fermi energy and thus are potentially of considerable interest in spin electronics. ${ }^{1-3}$ The proposal that there might exist such half-metallic ferromagnetic materials with $100 \%$ zero-temperature spin polarization at the Fermi level has quite a long history ${ }^{4-8}$ dating back to de Groot. ${ }^{5,6,8}$ Among the most cited candidates for $100 \%$ spin polarization are the semi-Heusler alloys (NiMnSb), ${ }^{5-12}$ "full" Heusler alloys, ${ }^{12-14}$ zinc-blende structure materials, ${ }^{15-17}$ colossal magnetoresistance materials $\left[\mathrm{La}_{1-x} \mathrm{Sr}_{x} \mathrm{MnO}_{3},{ }^{18,19} \mathrm{Sr}_{2} \mathrm{FeMoO}_{6}\right.$ (Ref. 20)], and semimetallic magnetic oxides $\left[\mathrm{CrO}_{2}\right.$ (Refs. 21-24) and $\mathrm{Fe}_{3} \mathrm{O}_{4}$ (Refs. 25 and 26)]. A number of other materials have also been suggested as half-metallic ferromagnets. ${ }^{4,27}$

Practical electronic devices that exploit the spin as well as the charge of the electron, ${ }^{28,29}$ including nonvolatile magnetic random-access memory elements, ${ }^{29,30}$ operate at nonzero temperatures, ideally at or above room temperature. Unfortunately, many potential half-metallic systems exhibit dramatic decreases in spin polarization and junction magnetoresistance well below room temperature, as discussed below. It is, therefore, necessary to consider finite temperature effects such as magnon and phonon excitations. Furthermore, if spin injection into a semiconductor, or spin-polarized tunneling is the goal, then the properties of the interface have to be considered, including the effects of finite temperatures on the electronic, magnonic, and phononic states at the interface and the quality of the interface. As we will elaborate (vide infra), these complications are common to all potential halfmetallic systems, so that the question arises whether these materials are indeed "operationally" better spin injectors than $\mathrm{Fe}(40 \%)$, Co $(34 \%)$, or even Ni $(23 \%)$.

To understand the effect of thermal excitations and reduced symmetries at surfaces and defects, one must take into account the localized features of the iron-series $3 d$ electrons, even in itinerant systems. In itinerant magnets, the $3 d$ electrons hop from atom to atom, and therefore contribute to the conductivity, but during their temporary residence on a given atomic site they adapt to the local environment. This is seen most clearly at finite temperatures, ${ }^{31}$ where spin disorder can be approximated by random intra-atomic exchange fields. ${ }^{32,33}$ Essentially, the effect of local disorder is to rotate the local spin direction and thus modify the local magnetic moment ${ }^{34}$ and spin polarization. ${ }^{35,36}$ The coupling between the atomic moments can then be understood in terms of Heisenberg-type exchange constants, and the signs and magnitudes of the constants determine, for example, whether the spin structure is collinear or noncollinear. In addition, there is a small ${ }^{34}$ noncollinearity due to spin-orbit coupling. ${ }^{35-38}$

The modification of the spin structures due to local perturbations is a relatively common phenomenon and easily rationalized in terms of a Landau-Ginzburg approach. ${ }^{34,39,40}$ The idea is to treat the atoms as a continuum in which exchange interactions are described by a gradient term. For example, at surfaces the approach yields an exponentially decaying perturbation. ${ }^{39,40}$ Surfaces, interfaces, defects, thermal disorder, and other aperiodicities have a pronounced effect on the spin polarization.

\section{SPIN DISORDER AND CONDUCTIVITY}

As noted now by several groups, ${ }^{41-44}$ spin disorder has a profound influence on the persistence of half-metallic character at finite temperature. Spin-mixing effects in ferromagnetic metals have been investigated for many years, for example, in the context of finite-temperature excitations in elemental nickel. ${ }^{32}$ A simple tight-binding description is provided by

$$
H_{\mu \nu i k \sigma \sigma^{\prime}}=T_{\mu \nu i k} \delta_{\alpha \beta}-V_{\mu} \delta_{\mu \nu} \delta_{i k} \delta_{\alpha \beta}-I_{o} \delta_{\mu \nu} \delta_{i k} \mathbf{e}_{i} \cdot \boldsymbol{\sigma}_{\alpha \beta},
$$

where $T_{\mu \nu i k}=\left\langle\phi_{\mu}\left(\mathbf{r}-\mathbf{R}_{i}\right)|T| \phi_{\nu}\left(\mathbf{r}-\mathbf{R}_{k}\right)\right\rangle$ is the hopping integral between a $\mu$-type orbital at $\mathbf{R}_{i}$ and a $\nu$-type orbital at $\mathbf{R}_{k}, \mathbf{V}_{\mu}$ is the crystal-field energy for the $\mu$-type orbital, $I_{o}$ is the intra-atomic $d$-electron exchange, and $\mathbf{e}_{i}$ is the local magnetization direction. In terms of Eq. (1), ferromagnetism results from the $I_{o}$ Stoner term, which reduces and enhances the energies of $\uparrow$ and $\downarrow$ electrons, respectively. 


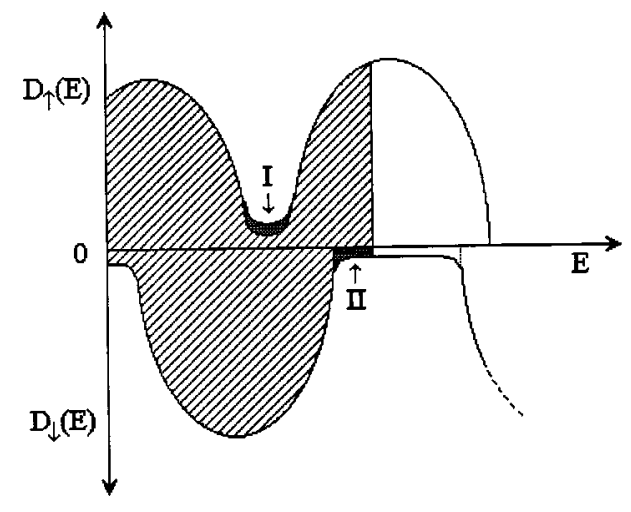

FIG. 1. Schematic $\uparrow$ and $\downarrow$ densities of states (dark shadowing denotes regions occupied at finite temperature only).

We have used Eq. (1) to model half-metallic ferromagnets ${ }^{41,42}$ and to compare with ordinary itinerant ferromagnets. As in other systems, ${ }^{32,33}$ the description of finitetemperature spin disorder involves the standard spin-1/2 rotation matrix $\hat{U}(\phi, \theta)$, which describes the rotation of a $\uparrow$ spin by the angles $\theta$ and $\phi$. The inverse matrix $\hat{U}^{-1}$ $=\hat{U}^{+}\left(\phi_{i}, \theta_{i}\right)$ diagonalizes the exchange term $\mathbf{e}_{i} \cdot \boldsymbol{\sigma}_{\alpha \beta}$ in Eq. (1), while applying the unitary matrix $\Pi_{i} \hat{U}^{+}\left(\phi_{i}, \theta_{i}\right)$ to Eq. (1) yields

$$
\begin{aligned}
H_{\mu \nu i k \alpha \beta}^{t}= & T_{\mu \nu i k} \Sigma_{\gamma} U_{\alpha \gamma}\left(\phi_{i}, \theta_{i}\right) U_{\gamma \beta}^{+}\left(\phi_{k}, \theta_{k}\right) \\
& -\left(V_{\mu} \delta_{\alpha \beta}+I_{o} \sigma_{z \alpha \beta}\right) \delta_{\mu \nu} \delta_{i k},
\end{aligned}
$$

where the thermal spin disorder has been mapped onto random hopping integrals.

Far below $T_{c}$, the densities of states (DOS) of ordinary ferromagnets are only weakly modified by thermal fluctuations, because $k_{\mathrm{B}} T$ is much smaller than the Fermi energy. However, the behavior of half-metallic ferromagnets cannot be reduced to a smearing of the Fermi level by a relative amount of the order of $k_{\mathrm{B}} T / E_{\mathrm{F}} \cdot{ }^{41-44}$ Figure 1 shows typical finite-temperature $\uparrow$ and $\downarrow$ densities of state. ${ }^{41,42}$ The distortion of the $\uparrow$ DOS, $D_{\uparrow}(E)$, has the character of a quantitative correction (dark region I), but in the $\downarrow$ gap the DOS changes qualitatively, from zero to nonzero (dark region II). This spin mixing yields nonzero $D_{\downarrow}(E)$ values in the middle of the spin-down gap, well above the highest energy level occupied at zero temperature. As a consequence, the resistance of the $\downarrow$ channel changes from infinity to a finite value.

The appearance of the spin-down density (II) in Fig. 1 has a simple physical interpretation: a $\uparrow$ electron hops onto a neighboring atomic site characterized by a different axis of spin quantization. In the frame of the neighboring atom, the spin wave function has nonzero projections onto both $\uparrow$ and $\downarrow$ states. For random disorder, the spin mixing can be interpreted as an Anderson localization of spin-polarized $3 d$ electrons in a thermally randomized atomic potential. In a very crude approximation, the magnitude of this spin-mixing contribution to the DOS is proportional to $M_{o}-M_{s}(T)$, where $M_{s}(T)$ is the spontaneous magnetization and $M_{o}=M_{s}(0){ }^{41,42}$ Considering a ferromagnet-insulatorferromagnet tunnel-junction geometry, Itoh, Ohsawa, and Inoue $^{43}$ have calculated the spin polarization at interfaces

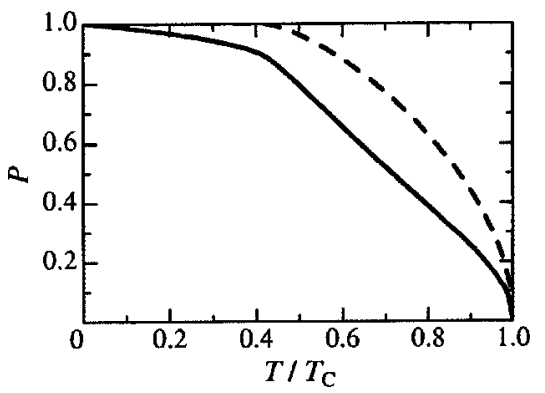

FIG. 2. The temperature dependence of the polarization $P$, either with (solid) and without (dashed) spin fluctuations for a half metal, adapted from Ref. 43.

with and without spin fluctuations considered. Figure 2 indicates that the effect of spin fluctuations is quite significant.

In Sec. III, we will see that the many-sublattice character of typical half metals modifies this picture. There is enhanced magnon and phonon coupling, with a suppression of $100 \%$ spin polarization at the Fermi level.

\section{MAGNONS AND PHONONS}

Magnon excitations lead to small decreases in polarization at low temperatures, more or less proportional to the drop in magnetization, but dramatic drops in polarization have been observed well below room temperature for many potential half-metallic systems. The tunnel magnetoresistance of $\mathrm{La}_{1-x} \mathrm{Sr}_{x} \mathrm{MnO}_{3} / \mathrm{SrTiO}_{3} / \mathrm{La}_{1-x} \mathrm{Sr}_{x} \mathrm{MnO}_{3}$ where $x$ $=0.2$ and $x=0.3$ dramatically drops at about $27 \mathrm{~K}$ (Ref. 45) and $130 \mathrm{~K}$ (Refs. 46-50), respectively. Dramatic drops in the measured polarization have been seen for $\mathrm{La}_{0.7} \mathrm{Ca}_{0.3} \mathrm{MnO}_{3},{ }^{51}$ $\mathrm{CrO}_{2},{ }^{52,53}$ and $\mathrm{NiMnSb} .{ }^{54-57}$ These decreases in polarization suggest that yet another temperature dependent mechanism for depolarization exists.

Gauging the magnitude of the spin polarization at the Fermi level and its pronounced drop well below room temperature, we must take into account the multisublattice character of potential half-metallic ferromagnets. ${ }^{41,42}$ For example, increasing the temperature of $\mathrm{NiMnSb}(100)$ beyond $80 \mathrm{~K}$ leads to pronounced changes in the band structure polarization, as probed by magnetic circular dichroism (MCD),${ }^{54}$ shown in Fig. 3 and summarized in Fig. 4. This is accompanied by a decrease in the $\langle 200\rangle$ neutron magnetic scattering factor with increasing temperature ${ }^{54,55}$ (inset of Fig. 4). This as well as both the differential magnetoresistance $\left[d(\Delta R / R) / d\left(\mu_{0} H\right)\right]$ [Fig. 4(a) $]$ and the resistance [Fig. 4(b)] of NiMnSb are consistent with a crossover in magnetic ordering at 80 to $100 \mathrm{~K}$. At temperatures above this metamagnetic transition, all indications suggest an onset of a large spin minority channel. ${ }^{54}$

Associated with the compound structure of all the postulated half metals, there are typically a number of lowenergy transverse and longitudinal optical modes. These optical phonon modes can couple to spin-wave modes and reduce the net magnetization. ${ }^{42}$ In real space, these magnetization modes correspond to a tilting between neighboring atomic spins, thus the tilt angle depends on the $k$ vector where the phonon and magnon bands cross. Generally, the 


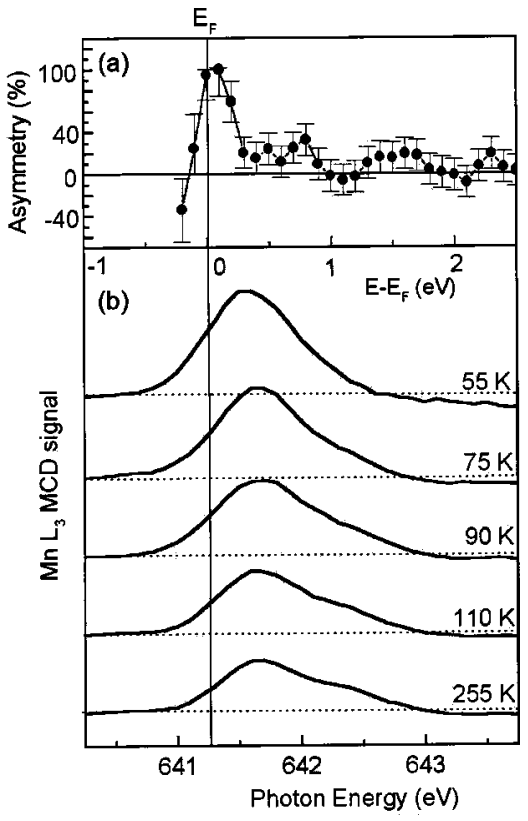

FIG. 3. The polarization asymmetry for $\mathrm{NiMnSb}(100)$ from spin-polarized inverse photoemission at the elevated temperature of $300 \mathrm{~K}$ (a) is compared to the temperature dependent magnetic circular dichroism signal data across Mn $2 p$ (b) core levels (Ref. 54). The MCD signal is shown for several different temperatures, increasing as the temperature decreases.

closer to the Brillouin zone edge the magnons and transverse optical modes couple, the greater the tilt angle. The corresponding moment misalignment gives rise to a pronounced spin mixing.

In the case of $\mathrm{NiMnSb}$, nearly dispersionless transverseand logitudinal optical modes are observed at about 28

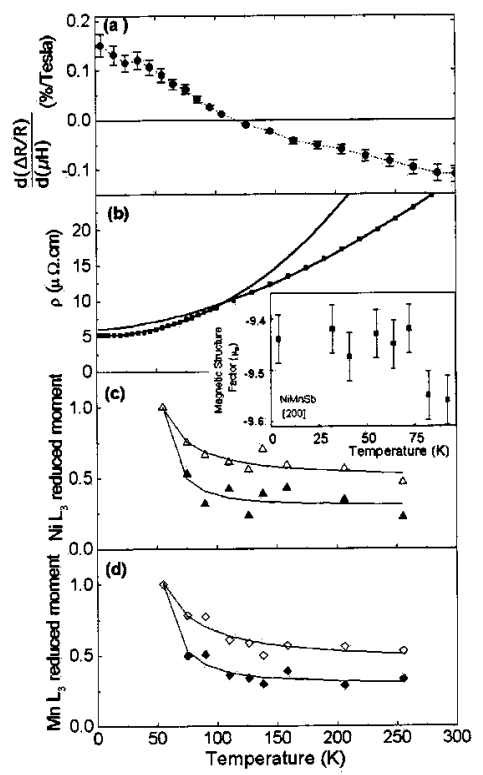

FIG. 4. Evidence of the metamagnetic transition in NiMnSb, taken from (Ref. 54), with permission. (a) Magnetoresistance slope $\left[d(\Delta R / R) / d\left(\mu_{0} H\right)\right]$, (b) the resistivity, (c) the relative Ni moment, and (d) relative $\mathrm{Mn}$ moment derived from MCD are compared. The change in the magnetic structure factor for the $\langle 200\rangle$ peak in neutron scattering shown as an inset, as adapted from (Ref. 55). The $T^{2}$ and $T^{1.65}$ curves are fitted to the resistance data.

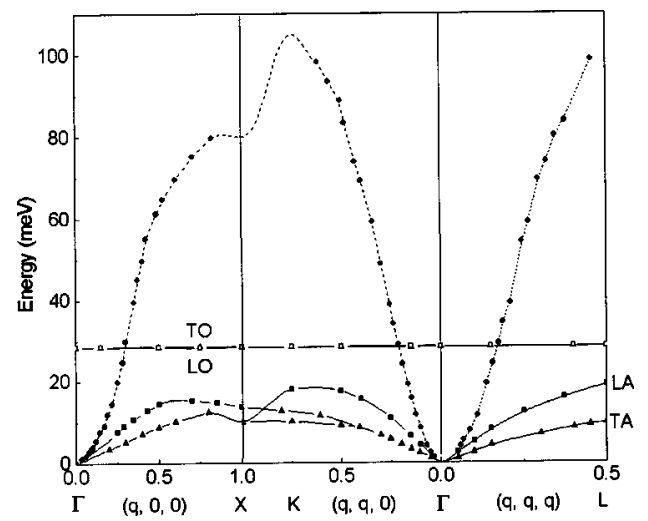

FIG. 5. The phonon and magnon band structure for NiMnSb taken from (Ref. 58). Dispersion curves for magnons $(\bullet)$, longitudinal phonons (ם), and transversal phonons $(\mathbf{\Delta})$ at $300 \mathrm{~K}$ are shown. No magnetic field was applied.

$\mathrm{meV},{ }^{58}$ as illustrated by the experimental phonon band structure reproduced in Fig. 5. Magnon coupling to these phonon modes should result a dramatic loss in Ni and Mn magnetization and polarization in NiMnSb, well below room temperature, as is observed. ${ }^{54}$ In the semi-Heusler alloys, this corresponds to a tilting between neighboring $\mathrm{Ni}$ and $\mathrm{Mn}$ atoms. In real space, the coupled magnon-phonon mode may cant the spins between Mn next nearest neighbors to form an angle of the order of $60^{\circ}$, while all the Mn moments are misaligned with respect to the nickel moments. ${ }^{54}$

Manganese perovskites exhibit a low-temperature spinwave softening and broadening of magnetoelastic origin, closely related to the proximity of the charge-ordered insulating state. ${ }^{59}$ The effect occurs where the magnon dispersion crosses the longitudinal optical branch of the phonons, at $k a / 2 \pi=0.3 .^{59,60}$ The lowest transverse optical modes in these systems, about $23 \mathrm{meV}$ for $\mathrm{La}_{0.7} A_{0.3} \mathrm{MnO}_{3}(A$ $=\mathrm{Ca}, \mathrm{Sr}),{ }^{59,60}$ suggest metamagnetic transitions should occur in the manganese perovskites, similar to those observed in NiMnSb, ${ }^{58}$ at about $84 \mathrm{~K}$. This suggests that the loss of net spin polarization should occur, and indeed there are indications this does occur, as noted above, at about 100-130 $\mathrm{K} .{ }^{46-50}$ The magnon phonon coupling is quite strong, ${ }^{61,62}$ leading to complete damping of the magnons from about $k a / 2 \pi \approx 0.3$ to the Brillouin zone boundary. ${ }^{59-61}$ Similar phonon modes exist in a variety of compounds, including materials such as $\mathrm{CrO}_{2}$ and $\mathrm{Fe}_{3} \mathrm{O}_{4}$. That phonon effects should be so profound should not be much of a surprise, given the existence of a charge ordering transition in the perovskites and the nonmetal to metal Verwey transition in $\mathrm{Fe}_{3} \mathrm{O}_{4}$.

Note that an elevated Curie temperature $T_{c}$ is not a good indicator of spin polarization at elevated temperatures. SemiHeusler alloys $A B C(A=\mathrm{Ni}, \mathrm{Pd}, \mathrm{Pt} ; B=\mathrm{Mn} ; C=\mathrm{P}, \mathrm{As}, \mathrm{Sb})$ with a $T_{c}$ of about $740 \mathrm{~K}$ have just as significant drops in polarization below room temperature as the manganese perovskites: $\mathrm{La}_{0.35} A_{0.65} \mathrm{MnO}_{3}(A=\mathrm{Sr}, \mathrm{Ba}, \mathrm{Pb})$ with a $T_{c}$ $=360 \mathrm{~K}$ or $\mathrm{CrO}_{2}$ with a $T_{c}=397 \mathrm{~K}$. First, from other manysublattice magnets, ${ }^{63}$ it is known that different sublattice magnetizations have different temperature dependencies. In 
small magnetic fields, of interest in the present context, this effect may be very pronounced, but high magnetic fields tend to align sublattices and smooth noncollinearities. This enhances the magnetization well below $T_{c}$. In extreme cases, the zero magnetic field magnetization curve $M(T)$ should indicate a magnetization far smaller than the magnetization curves for the same sample taken with moderate to high magnetic fields, for much of the temperature region below the ferromagnetic Curie temperature. A similar effect is encountered at interfaces. ${ }^{43}$ Second, different transition-metal sublattices yield, in general, different spin-scattering contributions per atom.

\section{THE PROBLEM OF INTERFACES}

It is very difficult to see how magnons can be eliminated from potential half-metallic systems to provide true halfmetal behavior. One could consider suppressing the long wavelength (and low-energy) magnons by breaking up the half-metallic ferromagnetic crystal into small crystallites, but this approach does not provide any advantage as even stronger spin-mixing effects are caused by surfaces and interfaces. Spin minority surface states, well known in half metals, $8,12,17,64-66$ can "develop" into interface states ${ }^{65-67}$ and therefore lead to the loss of half-metallic character. Indeed the creation of more interfaces presents a considerable problem as increases in interface density result in increases in the spin minority population decreasing the overall spin polarization as well, even near $0 \mathrm{~K}$.

For the stoichiometric surface, a surface electronic structure that differs from the bulk should not be surprising because there is experimental evidence of large surface enthalpy differences from the bulk in these materials. There are strong chemical potential for surface segregation in these materials ${ }^{68,69}$ indicating that the equilibrium surface is not the same stoichiometry as the bulk. ${ }^{68-73}$

\section{EXPERIMENTAL PROOF OF HALF-METALLIC FERROMAGNETISM}

There have been various experimental arguments in favor of true half-metallic ferromagnetism. ${ }^{1,2,446,74-82}$ Polarization measurements at very low temperature ${ }^{46,80-82}$ do not yield compelling evidence that there are no magnon contributions at low temperatures, even at $1.6 \mathrm{~K}$. For example, even though tunnel junctions include contributions from the spin dependent Fermi velocity, which could effectively enhance the tunnel magnetoresistance, ${ }^{83}$ no tunnel magnetoresistive junction measurement has provided a strong indication of half-metallic character. The highest tunnelmagnetoresistance values reached have been as high as $150 \%$ to $730 \%$ (Refs. $45,47,48$, and 51 ) to $1800 \%,{ }^{46}$ and this only at very low temperatures (though very high values of magnetoresistance have been observed at $77 \mathrm{~K},{ }^{51}$ and room temperature ${ }^{84}$ ). Unfortunately, interface and defect scattering contributions to the magnetoresistance are difficult to eliminate, making such measurements less than compelling. It is hard to see how magnon scattering can be eliminated from magnetoresistive junctions at small applied bias ${ }^{85}$ Unfortunately, indirect proof of half-metallic character from the
TABLE I. Experimental spin polarization determined by spin-polarized electron spectroscopies at elevated sample temperatures. With the exception of the spin-polarized inverse photoemission experiments in (Refs. 54, 71, 73, and 78), all data were obtained by spin-polarized photoemission.

\begin{tabular}{clc}
\hline \hline $\begin{array}{c}\text { Potential } \\
\text { half-metallic system }\end{array}$ & \multicolumn{1}{c}{ Polarization claim } & Temperature (K) \\
\hline $\mathrm{La}_{0.7} \mathrm{Sr}_{0.3} \mathrm{MnO}_{3}$ & $100 \pm 5 \%$ (Ref. 74) & 40 \\
$\mathrm{La}_{0.7} \mathrm{~Pb}_{0.3} \mathrm{MnO}_{3}$ & $100 \%$ (Ref. 78) & 100 \\
& Much less than 80\% & 300 \\
& (Ref. 71) & \\
$\mathrm{CrO}_{2}$ & 13\% (Ref. 100) & 10 \\
& $95 \%$ (Ref. 75) & 293 \\
$\mathrm{Fe}_{3} \mathrm{O}_{4}$ & $95 \%$ (Ref. 76) & 293 \\
& $-80 \pm 5 \%$ (Ref. 77) & 293 \\
$\mathrm{NiMnSb}$ & $-40 \%$ (Ref. 91) & 130 \\
& $50 \%$ (Ref. 92) & 300 \\
& $40 \%$ (Ref. 93) & 300 \\
& High but not near & 300 \\
\hline \hline
\end{tabular}

magnetic moment measured at high fields is also likely to be very insensitive to a small spin minority density of states.

Transport spin-polarization measurements, using pointcontact Andreev reflection, have generally provided the highest measured polarizations to date. ${ }^{80-82,86-90}$ In spite of several claims of proof of half-metallic character for $\mathrm{CrO}_{2}$, from point contact Andreev reflection, ${ }^{80,81}$ superconducting tunnel junctions ${ }^{82}$ and magneto-resistive transport ${ }^{52}$ measurements, these measured values of polarization $\left(96 \pm 1 \%,{ }^{80}\right.$ $98.4 \%,{ }^{81} 97 \%{ }^{82}, 82 \%,{ }^{52}$ respectively) generally miss the mark of $100 \%$ polarization. The analysis of the data must be taken with considerable care ${ }^{86,89}$ and cannot ignore Fermi velocity contributions. In the case of $\mathrm{La}_{2 / 3} \mathrm{Sr}_{1 / 3} \mathrm{MnO}_{3}$, the appreciable spin minority population at $1.5 \mathrm{~K}$ is probably a band-structure effect. ${ }^{86}$ High polarization combined with a large Fermi velocity in one spin channel, versus low polarization and large localization in the other spin channel, can accentuate an already high polarization in many transport measurements.

There have also been claims of $100 \%$ spin polarization at temperature well above zero kelvin. Apart from a somewhat ambiguous scanning tunneling microscopy spectroscopy study undertaken at $77 \mathrm{~K},{ }^{79}$ claims of $100 \%$ spin polarization at more elevated temperatures $(>40 \mathrm{~K})$ rest on evidence from spin-polarized photoemission ${ }^{75-77}$ and spinpolarized inverse photoemission, ${ }^{78}$ as summarized in Table I. Spin-resolved photoemission measurements ${ }^{75-77}$ and spinpolarized inverse photoemission ${ }^{78}$ that claim to provide evidence of half-metal behavior, in fact, do not. (Of course, not all such measurements of potential half-metallic systems have led to such claims. ${ }^{71,73,91-93}$ ) Finite temperature effects leading to the population of spin minority states near $E_{\mathrm{F}}$, as outlined in the previous sections, would be most significant at wave vectors away from $\bar{\Gamma}$. For this reason, they may not be observed by spin-polarized photoemission at normal emission $^{74-77}$ or by spin-polarized inverse photoemission at normal incidence, ${ }^{78}$ even for stoichiometric surfaces. Similarly, spin minority surface states leading to a loss of halfmetallic character, as noted above, would not generally be 
observed if measurements are only undertaken at the $\bar{\Gamma} k_{\|}$ $=0$ point.

Polarization at the Brillouin zone center $\left(\mathbf{k}_{\|}=0\right)$ corresponds to long range magnetic order but is only loosely related to the magnetic moment. Figure 3 illustrates this point for the polarization of the $\mathrm{NiMnSb}(100)$ surface. The data suggest a very high spin polarization near the Fermi level, nearly $100 \%$ above background for $\mathbf{k}_{\|}=0$ and $T=300 \mathrm{~K}$ (Refs. 54 and 73) in spin-polarized inverse photoemission, but as the temperature is increased above $80 \mathrm{~K}$, the MCD indicates a huge decrease in the relative band structure wave vector "averaged" polarization. Angle-resolved spinpolarized photoemission or spin-polarized inverse photoemission measurements, with limited wave vector sampling, cannot be taken as sufficient evidence of half-metallic ferromagnetic character if the sample is crystalline or is polycrystalline with texture growth.

The negligible DOS at the Fermi level $\left[D\left(E_{\mathrm{F}}\right) \sim 0\right]$ in the spin-polarized photoemission spectra of $\mathrm{La}_{2 / 3} \mathrm{Sr}_{1 / 3} \mathrm{MnO}_{3}$ (Ref. 74) and $\mathrm{CrO}_{2}$ (Ref. 75) and in spin-polarized inverse photoemission for $\mathrm{La}_{2 / 3} \mathrm{~Pb}_{1 / 3} \mathrm{MnO}_{3}$ (Ref. 71) suggests that either the surface is not the correct stoichiometry in those studies (see Ref. 70 for $\mathrm{La}_{2 / 3} \mathrm{Sr}_{1 / 3} \mathrm{MnO}_{3}$, Ref. 72 for $\mathrm{La}_{2 / 3} \mathrm{~Pb}_{1 / 3} \mathrm{MnO}_{3}$, Ref. 68 for NiMnSb, and Refs. 94 and 95 for $\mathrm{CrO}_{2}$ ) or that the Fermi level crossings are well away from $\bar{\Gamma}$. To a very small extent, final state effects in photoemission can also contribute. ${ }^{96} \mathrm{~A}$ band structure like that of gadolinium, with a strong magnetic surface state near the Fermi energy at $\bar{\Gamma}$, might appear to be a very high polarization ferromagnet, if such measurements are taken at only one wave vector near the surface Brillouin zone center, ${ }^{97-99}$ well below the Debye temperature. ${ }^{99}$ No one would argue that gadolinium is a half-metallic system. Possible crystalline disorder that might occur cannot be used as an argument for "complete" sampling of the Brillouin zone. In such strongly textured thin films, disorder would result in some cylindrical averaging of $k$ points away from $\bar{\Gamma}$ but leave the $\bar{\Gamma}$ point unperturbed. So "proof" of half-metallic character must necessarily remain elusive.

\section{CONCLUSIONS}

We have, hopefully, convinced the reader that $98 \%$ spin polarization is not $100 \%$ spin polarization, even at $1.6 \mathrm{~K}$. Magnons are difficult to suppress, in spite of a fond desire for $100 \%$ spin polarization at room temperature. We have shown that finite-temperature spin disorder destroys the complete spin polarization of half-metallic ferromagnets. The many-sublattice structure of the half-metallic ferromagnets, which is most pronounced in zero magnetic field conditions, yields transverse optical phonon modes which couple to thermally excited magnons. In a strict sense, this thermally activated spin mixing means that half-metallic ferromagnetism is limited to perfect crystals at zero temperature. This mechanism is modified and, in general, enhanced by crystal imperfections, and by surface and interface effects.

We do not equate the transport properties of half metals considered in this paper with those observed in ordinary itinerant ferromagnets. In the density of states, the difference is epitomized by Fig. 1, and the transport is influenced by the contrast between itinerant majority and largely localized minority electrons. In our opinion, half-metallic ferromagnetism is an idealized limit, realistic only in perfect crystals at zero temperature. Due to finite-temperature and surface effects, the elemental ferromagnets ( $\mathrm{Co}, \mathrm{Fe}$, and $\mathrm{Ni}$ ) may, in fact, be better for spin injection than half-metallic systems.

\section{ACKNOWLEDGMENTS}

The support of the Office of Naval Research, the NSF "QSPINS" MRSEC (DMR 0213808), and the Nebraska Research Initiative is gratefully acknowledged. The authors also acknowledge comments and contributions from A. N. Caruso, H.-K. Jeong, T. Komesu, C. Borca, D. Ristoiu, J. P. Nozières, J. Pierre, J. Zhang, and E. W. Plummer.

${ }^{1}$ W. E. Pickett and J. Moodera, Phys. Today 54, 39 (2001).

${ }^{2}$ J. M. D. Coey and C. L. Chien, MRS Bull. 28, 724 (2003).

${ }^{3}$ M. Ziese, Rep. Prog. Phys. 65, 143 (2002).

${ }^{4}$ V. Yu. Irkhin and M. I. Katsnel'son, Phys. Usp. 37, 659 (1994).

${ }^{5}$ R. A. de Groot, F. M. Mueller, P. G. van Engen, and K. H. J. Buschow, Phys. Rev. Lett. 50, 2024 (1983).

${ }^{6}$ R. A. de Groot, F. M. Mueller, P. G. van Engen, and K. H. J. Buschow, J. Appl. Phys. 55, 2151 (1984).

${ }^{7}$ J. Kübler, Physica B 127, 257 (1984)

${ }^{8}$ C. M. Fang, G. A. de Wijs, and R. A. de Groot, J. Appl. Phys. 91, 8340 (2002).

${ }^{9}$ K. E. H. M. Hanssen and P. E. Mijnarends, Phys. Rev. B 34, 5009 (1986).

${ }^{10}$ I. Galanakis, S. Ostanin, M. Alouani, H. Dreysse, and J. M. Wills, Phys. Rev. B 61, 4093 (2000)

${ }^{11}$ J.-S. Kang, J.-G. Park, C. G. Olson, S. J. Youn, and B. I. Min, J. Phys.: Condens. Matter 7, 3789 (1995).

${ }^{12}$ I. Galanakis, J. Phys.: Condens. Matter 14, 6329 (2002).

${ }^{13}$ I. Galanakis, P. H. Dederichs, and N. Papanikolaou, Phys. Rev. B 66, 174429 (2002).

${ }^{14}$ I. Galanakis, P. H. Dederichs, and N. Papanikolaou, Phys. Rev. B 66, 134428 (2002).

${ }^{15}$ B. G. Liu, Phys. Rev. B 67, 172411 (2003).

${ }^{16}$ W. H. Xie, Y. Q. Xu, B. G. Liu, and D. G. Pettifor, Phys. Rev. Lett. 91, 037204 (2003).

${ }^{17}$ I. Galanakis, Phys. Rev. B 66, 012406 (2002).

${ }^{18}$ W. E. Pickett and D. J. Singh, Phys. Rev. B 53, 1146 (1996).

${ }^{19}$ D. J. Singh and W. E. Pickett, Phys. Rev. B 57, 88 (1998).

${ }^{20}$ K.-I. Kobayashi, T. Kimura, H. Sawada, K. Terakura, and Y. Tokura, Nature (London) 395, 677 (1998).

${ }^{21}$ K. Schwarz, J. Phys. F: Met. Phys. 16, L211 (1986).

${ }^{22}$ H. van Lueken and R. A. de Groot, Phys. Rev. B 51, 7176 (1995).

${ }^{23}$ M. A. Korotin, V. I. Anisimov, D. I. Khomskii, and G. A. Sawatzky, Phys. Rev. Lett. 80, 4305 (1998).

${ }^{24}$ S. P. Lewis, P. B. Allen, and T. Sasaki, Phys. Rev. B 55, 10253 (1997).

${ }^{25}$ R. A. de Groot and K. H. J. Bischow, J. Magn. Magn. Mater. 54-57, 1377 (1986).

${ }^{26}$ M. Penicaud, B. Silberchoit, C. B. Sommers, and J. Kubler, J. Magn. Magn. Mater. 103, 212 (1992).

${ }^{27}$ I. I. Mazin, Appl. Phys. Lett. 77, 3000 (2000).

${ }^{28}$ G. A. Prinz, Science 282, 1660 (1998).

${ }^{29}$ S. A. Wolf, D. D. Awschalom, R. A. Buhrman, J. M. Daughton, S. von Molnar, M. L. Roukes, A. Y. Chtchelkanova, and D. M. Tregar, Science 294, 1488 (2001).

${ }^{30}$ A. Cho, Science 296, 249 (2002).

${ }^{31}$ P. Fulde, Electron Correlations in Molecules and Solids (Springer, Berlin, 1991).

${ }^{32}$ E. M. Haines, V. Heine, and A. Ziegler, J. Phys. F: Met. Phys. 16, 1343 (1986).

${ }^{33}$ V. A. Gubanov, A. I. Liechtenstein, and A. V. Postnikov, Magnetism and the Electronic Structure of Crystals (Springer, Berlin, 1992).

${ }^{34}$ R. Skomski, J. Phys.: Condens. Matter 15, R841 (2003).

${ }^{35}$ D. Orgassa, H. Fujiwara, T. C. Schulthess, and W. H. Butler, Phys. Rev. B 60, 13237 (1999). 
${ }^{36}$ D. Orgassa, H. Fujiwara, T. C. Schulthess, and W. H. Butler, J. Appl. Phys. 87, 5870 (2000)

${ }^{37}$ L. M. Sandratskii, Adv. Phys. 47, 91 (1998).

${ }^{38}$ L. M. Sandratskii, Phys. Rev. B 64, 134402 (2001).

${ }^{39}$ P. A. Dowben, C. Waldfried, and R. Skomski, J. Phys.: Condens. Matter 10, 5833 (1998).

${ }^{40}$ A. Miller and P. A. Dowben, J. Phys.: Condens. Matter 5, 5459 (1993).

${ }^{41}$ R. Skomski and P. A. Dowben, Europhys. Lett. 58, 544 (2002).

${ }^{42}$ P. A. Dowben and R. Skomski, J. Appl. Phys. 93, 7948 (2003).

${ }^{43}$ H. Itoh, T. Ohsawa, and J. Inoue, Phys. Rev. Lett. 84, 2501 (2000).

${ }^{44}$ A. H. MacDonald, T. Jungwirth, and M. Kasner, Phys. Rev. Lett. 81, 705 (1998).

${ }^{45}$ T. Obata, T. Manako, Y. Shimakawa, and Y. Kubo, Appl. Phys. Lett. 74, 290 (1999).

${ }^{46}$ M. Bowen, M. Bibes, A. Barthélémy, J.-P. Contour, A. Anane, Y. Lemaître, and A. Fert, Appl. Phys. Lett. 82, 233 (2003).

${ }^{47}$ J. Z. Sun, W. J. Gallagher, P. R. Duncombe, L. Krusin-Elbaum, R. A. Altman, A. Gupta, Yu Lu, G. Q. Gong, and Gang Xiao, Appl. Phys. Lett. 69, 3266 (1996).

${ }^{48}$ M. Viret, J. Nassar, M. Drouet, J. P. Contour, C. Fermon, and A. Fert, J. Magn. Magn. Mater. 198-199, 1 (1999).

${ }^{49}$ J. Z. Sun, Philos. Trans. R. Soc. London, Ser. A 356, 1693 (1998).

${ }^{50} \mathrm{Yu}$ Lu, X. W. Li, G. Q. Gong, Gang Xiao, A. Gupta, P. Lecoeur, J. Z. Sun, Y. Y. Wang, and V. P. Dravid, Phys. Rev. B 54, R8357 (1996).

${ }^{51}$ M. H. Jo, N. D. Mathur, N. K. Todd, and M. G. Blamire, Phys. Rev. B 61, 14905 (2000).

${ }^{52}$ J. M. D. Coey and M. Venkatesan, J. Appl. Phys. 91, 8345 (2002).

${ }^{53}$ S. M. Watts, S. Wirth, S. von Molnár, A. Barry, and J. M. D. Coey, Phys. Rev. B 61, 9621 (2000).

${ }^{54}$ C. N. Borca, T. Komesu, H. Jeong, P. A. Dowben, D. Ristoiu, Ch. Hordequin, J. P. Nozières, J. Pierre, S. Stadler, and Y. U. Idzerda, Phys. Rev. B 64, 052409 (2001).

${ }^{55}$ Ch. Hordequin, E. Lelievre-Berna, and J. Pierre, Physica B 234-236, 602 (1997).

${ }^{56}$ Ch. Hordequin, D. Ristoiu, L. Ranno, and J. Pierre, Eur. Phys. J. B 16, 287 (2000).

${ }^{57}$ C. T. Tanaka, J. Nowak, and J. S. Moodera, J. Appl. Phys. 81, 5515 (1997).

${ }^{58}$ C. N. Borca, D. Ristoiu, T. Komesu, H. Jeong, Ch. Hordequin, J. Pierre, J. P. Nozières, and P. A. Dowben, Appl. Phys. Lett. 77, 88 (2000).

${ }^{59}$ P. Dai, H. Y. Hwang, J. Zhang, A. Fernandez-Baca, S.-W. Cheong, C. Kloc, Y. Tomioka, and Y. Tokura, Phys. Rev. B 61, 9553 (2000).

${ }^{60}$ J. Zhang, P. Dai, A. Fernandez-Baca, E. W. Plummer, Y. Tomioka, and Y. Tokura, Phys. Rev. Lett. 86, 3823 (2001).

${ }^{61}$ X. D. Wang, Phys. Rev. B 57, 7427 (1998).

${ }^{62}$ L. M. Woods, Phys. Rev. B 65, 014409 (2000).

${ }^{63}$ R. Skomski and J. M. D. Coey, Permanent Magnetism (Institute of Physics, Bristol, 1999).

${ }^{64}$ S. J. Jenkins and D. A. King, Surf. Sci. Lett. 494, L793 (2001).

${ }^{65}$ G. A. de Wijs and R. A. de Groot, Phys. Rev. B 64, 020402 (2001).

${ }^{66}$ S. J. Jenkins and D. A. King, Surf. Sci. Lett. 501, L185 (2002).

${ }^{67}$ S. Picozzi, A. Continenza, and A. J. Freeman, J. Phys. Chem. Solids 64, 1697 (2003)

${ }^{68}$ D. Ristoiu, J. P. Nozières, C. N. Borca, B. Borca, and P. A. Dowben, Appl. Phys. Lett. 76, 2349 (2000).

${ }^{69}$ J. Choi, J. Zhang, S.-H. Liou, P. A. Dowben, and E. W. Plummer, Phys. Rev. B 59, 13453 (1999).

${ }^{70}$ H. Dulli, E. W. Plummer, P. A. Dowben, Jaewu Choi, and S.-H. Liou, Appl. Phys. Lett. 77, 570 (2000).
${ }^{71}$ C. N. Borca, B. Xu, T. Komesu, H.-K. Jeong, M. T. Liu, S.-H. Liou, S. Stadler, Y. Idzerda, and P. A. Dowben, Europhys. Lett. 56, 722 (2001).

${ }^{72}$ C. N. Borca, B. Xu, T. Komesu, H.-K. Jeong, M. T. Liu, S.-H. Liou, and P. A. Dowben, Surf. Sci. Lett. 512, L346 (2002).

${ }^{73}$ D. Ristoiu, J. P. Nozières, C. N. Borca, T. Komesu, H.-K. Jeong, and P. A. Dowben, Europhys. Lett. 49, 624 (2000).

${ }^{74}$ J.-H. Park, E. Vescovo, H.-J. Kim, C. Kwon, R. Ramesh, and T. Venkatesan, Nature (London) 392, 794 (1998).

${ }^{75}$ K. P. Kämper, W. Schmitt, G. Güntherodt, R. J. Gambino, and R. Ruf, Phys. Rev. Lett. 59, 2788 (1987).

${ }^{76}$ Y. S. Dedkov, M. Fonine, C. Konig, U. Rüdiger, G. Güntherodt, S. Senz, and D. Hesse, Appl. Phys. Lett. 80, 4181 (2002).

${ }^{77}$ Y. S. Dedkov, U. Rüdiger, and G. Güntherodt, Phys. Rev. B 65, 064417 (2002).

${ }^{78}$ R. Bertacco, M. Portalupi, M. Marcon, L. Duo, F. Ciccacci, M. Bowen, J. P. Contour, and A. Barthélémy, J. Magn. Magn. Mater. 242-245, 710 (2002).

${ }^{79}$ J. Y. T. Wei, N. C. Yeh, and R. P. Vasquez, Phys. Rev. Lett. 79, 5150 (1997)

${ }^{80}$ Y. Ji, G. J. Strijkers, F. Y. Yang, C. L. Chien, J. M. Byers, A. Anguelouch, G. Xiao, and A. Gupta, Phys. Rev. Lett. 86, 5585 (2001).

${ }^{81}$ A. Anguelouch, A. Gupta, G. Xiao, G. X. Miao, D. W. Abraham, S. Ingvarsson, Y. Ji, and C. L. Chien, J. Appl. Phys. 91, 7140 (2002).

${ }^{82}$ J. S. Parker, S. M. Watts, P. G. Ivanov, and P. Xiong, Phys. Rev. Lett. 88, 196601 (2002)

${ }^{83}$ I. I. Mazin, Phys. Rev. Lett. 83, 1427 (1999).

${ }^{84}$ J. J. Versluijs, M. A. Bari, and J. M. D. Coey, Phys. Rev. Lett. 87, 026601 (2001).

${ }^{85}$ A. M. Bratkovsky, Appl. Phys. Lett. 72, 2334 (1998).

${ }^{86}$ B. Nadgorny, I. I. Mazin, M. Osofsky, R. J. Soulen, P. Broussard, R. M. Stroud, D. J. Singh, V. G. Harris, A. Arsenov, and Ya. Mukovskii, Phys. Rev. B 63, 184433 (2001).

${ }^{87}$ R. J. Soulen, J. M. Byers, M. S. Osofsky, B. Nadgorny, T. Ambrose, S. F. Cheng, P. R. Broussard, C. T. Tanaka, J. Nowak, J. S. Moodera, A. Barry, and J. M. D. Coey, Science 282, 85 (1998).

${ }^{88}$ W. J. DeSisto, P. R. Broussard, T. F. Ambrose, B. E. Nadgorny, and M. S. Osofsky, Appl. Phys. Lett. 76, 3789 (2000).

${ }^{89}$ I. I. Mazin, A. A. Golubov, and B. Nagorny, J. Appl. Phys. 89, 7576 (2001)

${ }^{90}$ Y. Ji, G. J. Strijkers, R. Y. Yang, and C. L. Chien, Phys. Rev. B 64, 224425 (2001).

${ }^{91}$ S. A. Morton, G. D. Waddill, S. Kim, I. K. Schuller, S. A. Chambers, and J. G. Tobin, Surf. Sci. 513, L451 (2002).

${ }^{92}$ G. L. Bona, F. Meier, M. Taborelli, E. Bucher, and P. H. Schmidt, Solid State Commun. 56, 391 (1985).

${ }^{93}$ W. Zhu, B. Sincovic, E. Vescovo, C. Tanaka, and J. S. Moodera, Phys. Rev. B 64, 060403(R) (2001).

${ }^{94}$ J. Dai, J. Tang, H. Xu, L. Spinu, W. Wang, K.-Y. Wang, A. Kumbhar, M. Li, and U. Diebold, Appl. Phys. Lett. 77, 2840 (2000).

${ }^{95}$ R. Cheng, B. Xu, C. N. Borca, A. Sokolov, C.-S. Yang, L. Yuan, S.-H. Liou, B. Doudin, and P. A. Dowben, Appl. Phys. Lett. 79, 3122 (2001).

${ }^{96}$ R. Joynt, Science 284, 777 (1999).

${ }^{97}$ I. N. Yakovkin, T. Komesu, and P. A. Dowben, Phys. Rev. B 66, 035406 (2002).

${ }^{98}$ R. Q. Wu and A. J. Freeman, J. Magn. Magn. Mater. 99, 81 (1991).

${ }^{99}$ K. Maiti, M. C. Malagoli, A. Dallmeyer, and C. Carbone, Phys. Rev. Lett. 88, 167205 (2002)

${ }^{100}$ S. F. Alvarado, W. Eib, P. Mutz, H. C. Sigmann, M. Capagna, and J. P. Remelka, Phys. Rev. B 13, 4918 (1976) 